\title{
Ultrasound for Critical Care Physicians: A Pericardial Effusion of Uncertain Significance
}

\section{Brandon Murguia, MD}

\author{
Department of Medicine \\ University of New Mexico School of Medicine \\ Albuquerque, NM USA
}

A 75-year-old woman with known systolic congestive heart failure (ejection fraction of $40 \%$ ), chronic atrial fibrillation on rivaroxaban oral anticoagulation, morbid obesity, and chronic kidney disease stage 3, was transferred to the Medical Intensive Care Unit for acute hypoxic respiratory failure thought to be secondary to worsening pneumonia.

She had presented to the emergency department 3 days prior with shortness of breath, malaise, left-sided chest pain, and mildly-productive cough over a period of 4 days. She had mild tachycardia on presentation, but was normotensive without tachypnea, hypoxia, or fever. Routine labs were remarkable for a leukocytosis of 15,000 cells/ $\mathrm{LL}$. Cardiac biomarkers were normal, and electrocardiogram demonstrated atrial fibrillation with rapid ventricular rate of $114 \mathrm{bpm}$. Chest $\mathrm{x}$-ray revealed cardiomegaly and left lower lobe consolidation consistent with bacterial pneumonia. Patient was admitted to the floor for intravenous antibiotics, cardiac monitoring, and judicious isotonic fluids if needed.

On night 2 of hospitalization, the patient developed respiratory distress with tachypnea, pulse oximetry of $80-85 \%$, and increased ventricular response into the $140 \mathrm{bpm}$ range. The patient remained normotensive. A portable anterior-posterior chest x-ray showed cardiomegaly and now complete opacification of the left lower lobe. She was transferred to the MICU for suspected worsening pneumonia and congestive heart failure.

Upon arrival to the intensive care unit, vital signs were unchanged and high-flow nasal cannula was started at 6 liters per minute. A focused point-of-care cardiac ultrasound (PCU) was done, limited in quality by patient body habitus, but nonetheless demonstrating the clear presence of a moderate pericardial effusion on subcostal long axis view. 


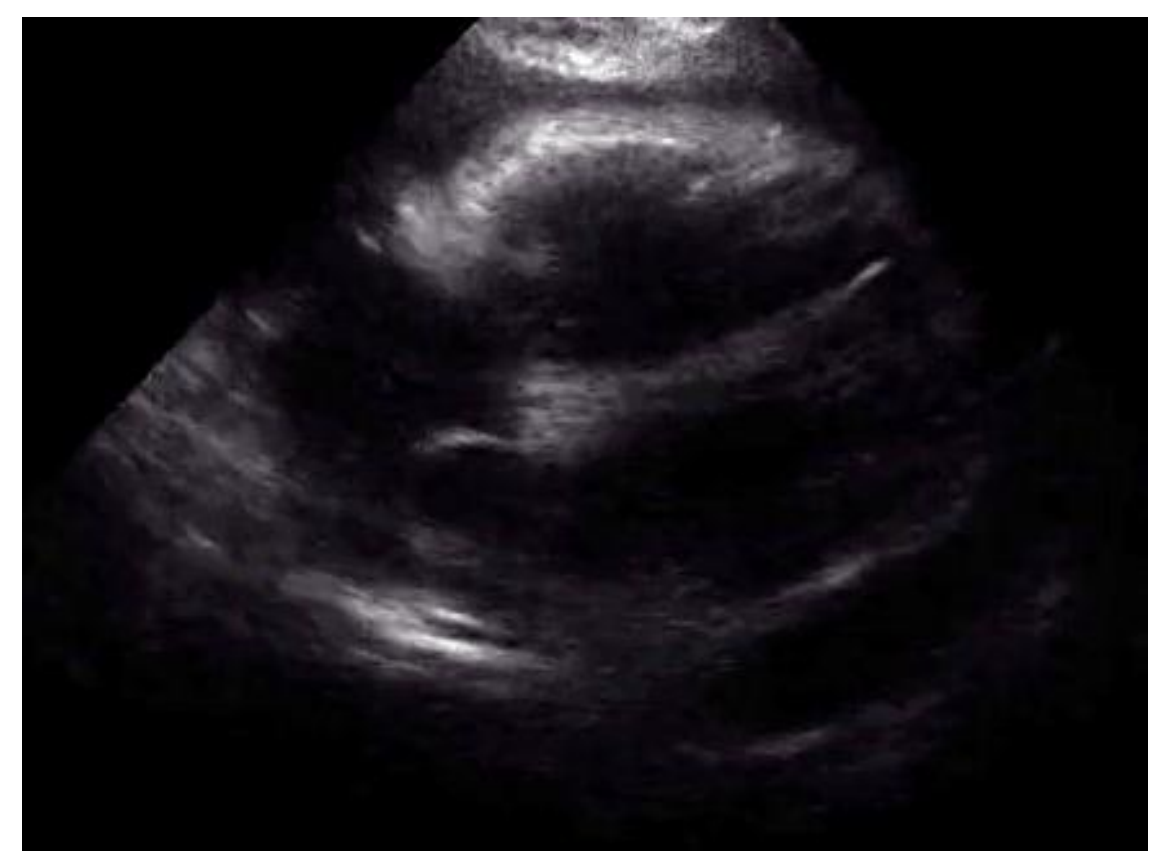

Figure 1: Subcostal long axis view of the heart.

What should be done next regarding this pericardial effusion?

1. Observe, this is not significant.

2. Additional echocardiographic imaging /evaluation.

3. Immediate pericardiocentesis.

4. Fluid challenge. 


\section{Correct! \\ 2. Additional echocardiographic imaging /evaluation.}

Early detection and management of cardiac tamponade is an essential skill in emergency and critical care medicine. Cardiac tamponade can be quickly and accurately identified by bedside point of care ultrasound (PCU). Physical exam findings, EKG, and chest x-ray abnormalities are less sensitive and specific than 2D echo.

While all of the above choices should be employed during initial PCU, tamponade physiology is most readily and accurately identified by right ventricular (RV) diastolic collapse in M-mode. IVC plethora with blunted collapsibility is very sensitive, but notoriously nonspecific for cardiac tamponade. Of note, transvalvular Doppler inflow velocities, or flow velocity paradoxus, is also accurate to diagnose tamponade but is less specific than diastolic collapse in M-Mode, and considerably more challenging in image acquisition and skill.

Incorporation of both the right ventricle and mitral valve anterior leaflet across the parasternal (or in this case, subcostal) axis in M-mode allows the provider to reliably detect right ventricular wall collapse during the diastolic phase of the cardiac cycle (i.e., the phase between mitral valve opening and closure).

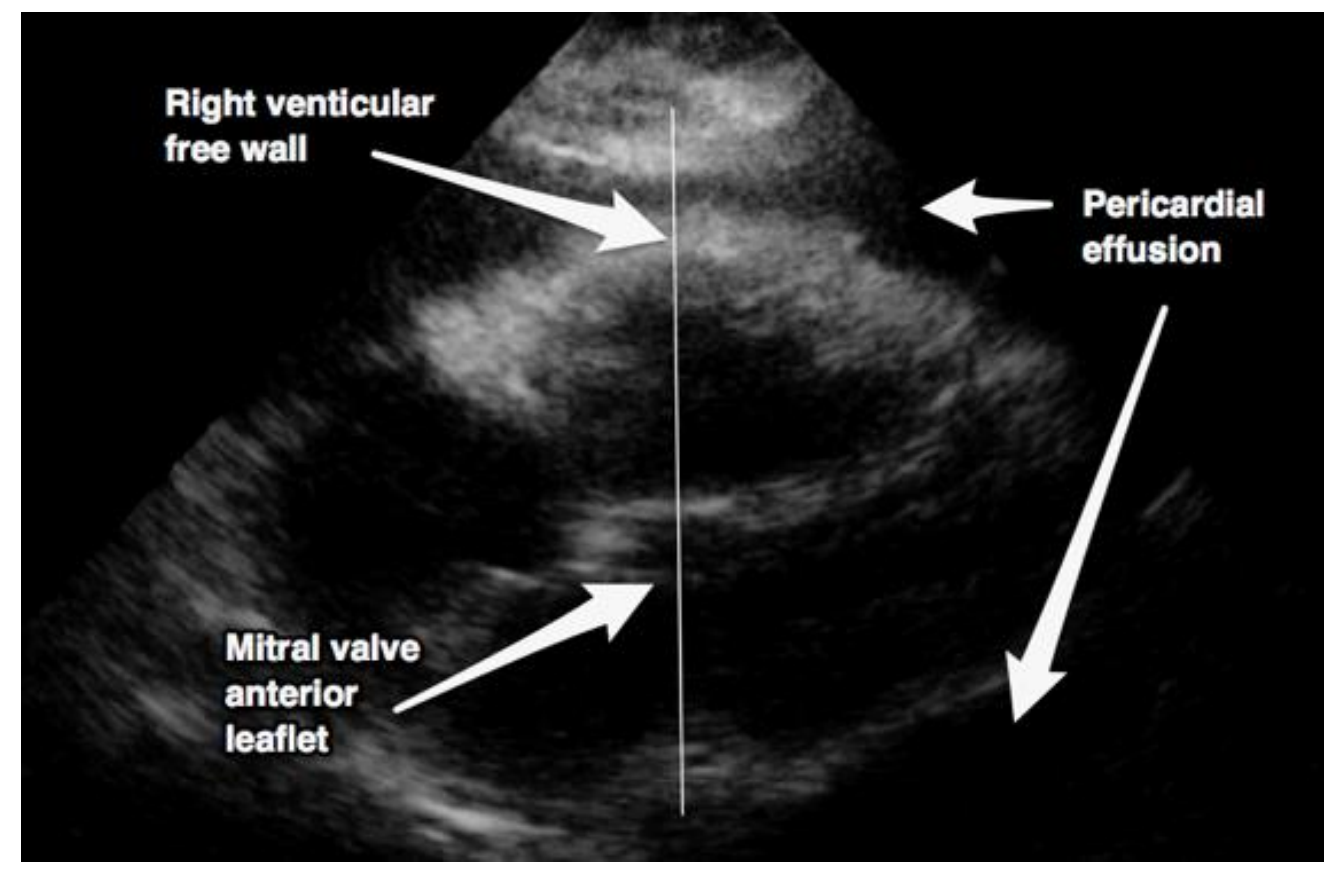

Figure 2. Demonstration of landmarks for M-Mode.

With M-mode, any posterior motion of the right ventricle free wall during mitral valve opening (demonstrated by anterior motion of anterior mitral valve leaflet) is recognized as chamber collapse and tamponade physiology, which was indeed the case in this patient (See Figure 3). Preparation for US-guided pericardiocentesis was planned and cardiology consulted. 


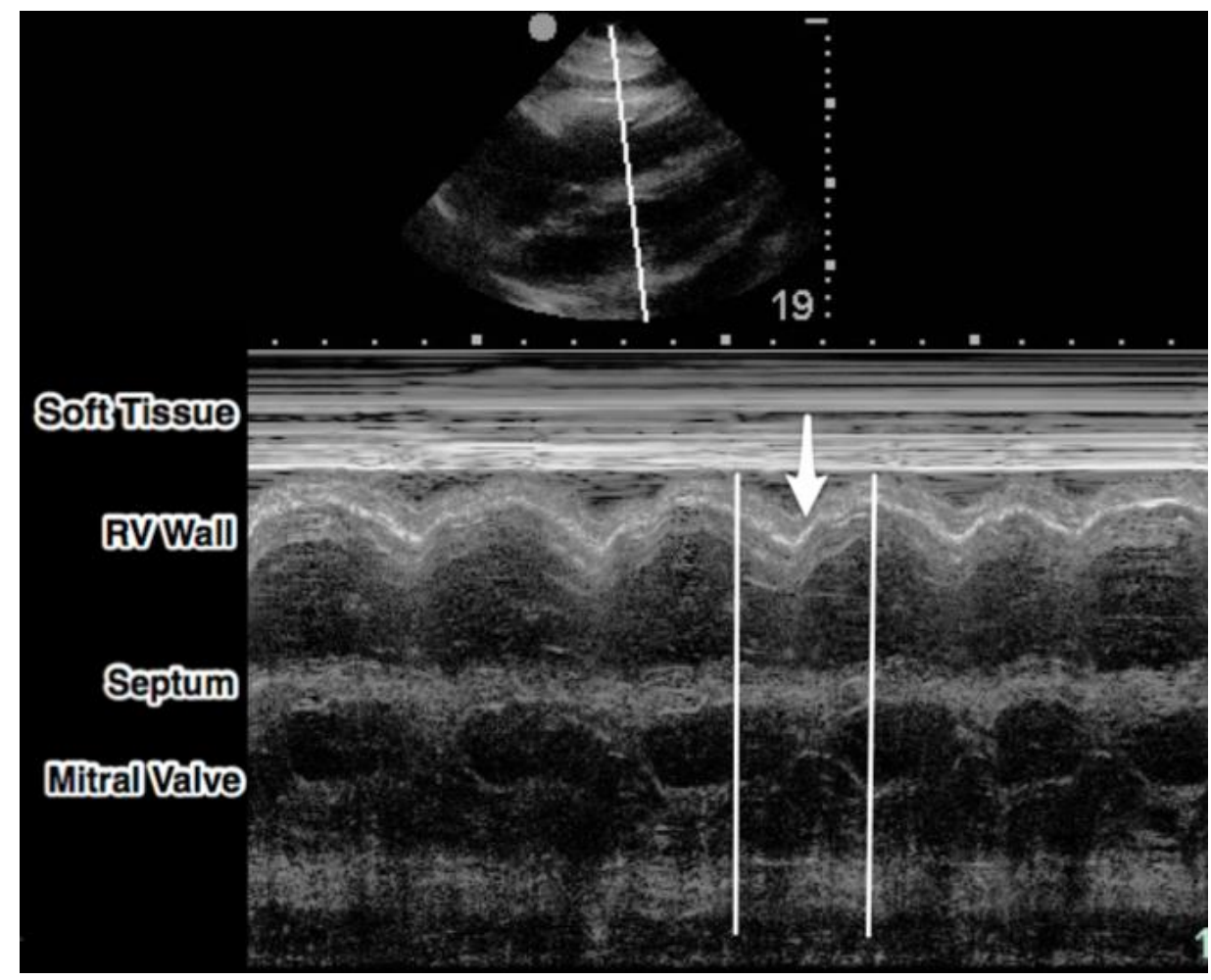

Figure 3. M-Mode view through RV free wall and Mitral valve. RV free wall collapse occurs during mitral valve opening in M-Mode. White arrow demonstrates posterior wall movement of RV Wall during diastole.

Formal STAT Echo read was obtained 1 hour later, confirming tamponade physiology. Pericardiocentesis was then successfully performed via para-apical approach under US guidance, with a large serosanguinous pericardial effusion (>600 ml) aspirated. Within minutes, the patient's tachycardia improved and her respiratory distress resolved, confirming the diagnosis of tamponade. Fluid studies demonstrated a hemorrhagic pericardial effusion with negative cultures and cytology. Her rivaroxaban was discontinued, a pericardial catheter kept in place until drainage of $<50 \mathrm{ml} / \mathrm{day}$, and antibiotics administered for the bacterial pneumonia. The patient was transferred back to the floor in stable condition 3 days later.

Final diagnosis: acute hypoxic respiratory failure secondary to early hemorrhagic pericardial tamponade in setting of rivaroxaban for chronic atrial fibrillation.

Take home point: pericardial effusion with tamponade occurs along a spectrum of widely-variable clinical presentations (more often silently than catastrophically in medical patients). While PCU can easily identify the presence or absence of a pericardial effusion, M-mode visualization of right ventricular free wall collapse during diastole should also be examined to quickly and accurately identify early tamponade physiology. 


\section{References}

1. Jacob S, Sebastian JC, Cherian PK, Abraham A, John SK. Pericardial effusion impending tamponade: a look beyond Beck's triad. Am J Emerg Med. 2009 Feb;27(2):216-9. [CrossRef] [PubMed]

2. Levine MJ, Lorell BH, Diver DJ, Come PC. Implications of echocardiographically assisted diagnosis of pericardial tamponade in contemporary medical patients: detection before hemodynamic embarrassment. J Am Coll Cardiol. 1991 Jan;17(1):59-65. [CrossRef] [PubMed]

3. Atar S, Chiu J, Forrester JS, Siegel RJ. Bloody pericardial effusion in patients with cardiac tamponade: is the cause cancerous, tuberculous, or iatrogenic in the 1990s? Chest. 1999 Dec;116(6):1564-9. [CrossRef] [PubMed]

4. Reydel B, Spodick DH. Frequency and significance of chamber collapses during cardiac tamponade. Am Heart J. 1990 May;119(5):1160-3. [CrossRef] [PubMed]

5. Nagdev A, Stone MB. Point-of-care ultrasound evaluation of pericardial effusions: does this patient have cardiac tamponade? Resuscitation. 2011 Jun;82(6):671-3. [CrossRef] [PubMed]

6. Spodick DH. Acute cardiac tamponade. N Engl J Med. 2003 Aug 14;349(7):684-90. [CrossRef] [PubMed] 